\section{Agenda AERO 2004}

\section{3-24 septiembre}

Controversias en Oncología, Murcia

Miembros AERO implicados: Dr. Manuel de las Heras, presidente de AERO (Hospital Universitario Virgen de la Arrixaca, Murcia)

\section{8 o 29 septiembre}

Curso de AERO sobre la Gestión de Habilidades Directivas en Barcelona - NH Calderón (Barcelona) Miembros AERO implicados: Dr. Alberto Biete, miembro asociado de AERO (Hospital Clínico Universitario de Barcelona), Dr. José Pardo, presidente asesor de AERO (Hospital General de Catalunya)

\section{3 al 7 de octubre}

Congreso de la ASTRO en Atlanta

Miembros AERO implicados: Dr. Jordi Giralt, secretario de la Junta de AERO (Hospital General de la Vall d'Hebrón, Barcelona), Dra. Laura Cerezo, vocal de la Junta de AERO (Hospital de la Princesa, Madrid) y Dra. Eloisa Bayo, tesorera de la Junta de AERO (Hospital Juan Ramón Jiménez, Huelva)

\section{8 de octubre}

Jornadas Oncológicas de Pediatría, Barcelona Miembros AERO implicados: Dr. Jordi Giralt, secretario de la Junta de AERO (Hospital General de la Vall d'Hebrón, Barcelona)

\section{3 al 15 de octubre}

Curso de AERO sobre Radioquimioterapia, Elche Miembros AERO implicados: Dr. Manuel de las Heras, presidente de AERO (Hospital Universitario Virgen de la Arrixaca, Murcia)

\section{0 - 22 de octubre}

Curso de Indicaciones Clínicas de la Oncología Radioterápica, Benasque

Miembros AERO implicados: Dr. José Antonio Carceller, Hospital Universitario de Lleida Arnau de Vilanova y miembro asociacido de AERO

\section{1-22 de octubre}

Congreso Sociedad Andaluza de Oncología en Sevilla Miembros AERO implicados: Dra Eloisa Bayo, tesorera de la Junta de AERO (Hospital Juan Ramón Jiménez, Huelva)

\section{4-28 de octubre}

23rd Annual ESTRO Meeting en Amsterdam

\section{1-12 de noviembre}

Jornadas de revisión post ESTRO, Sevilla

Miembros AERO implicados: Dra Eloisa Bayo, tesorera de la Junta de AERO (Hospital Juan Ramón Jiménez, Huelva) y Dr. Herruzo (organizadores)

\section{6 al 19 de noviembre}

FESEO, Valencia

Miembros AERO implicados: Dr. Manuel de las Heras, presidente de AERO (Hospital Universitario Virgen de la Arrixaca, Murcia)

19 al 20 de noviembre EORTC, Organización Europea para la Investigación y Tratamiento del Cáncer

Barcelona - Subcomité de tumores de cabeza y cuello Miembros AERO implicados: Dr. Jordi Giralt secretario de la Junta de AERO (Hospital General de la Vall d'Hebrón, Barcelona)

\section{2 al 24 de noviembre}

Curso AERO de Braquiterapia

Miembros AERO implicados: Dra. Ana Mañas, vicepresidenta de AERO (Hospital Universitario 12 de Octubre, Madrid)

\section{4 - 5 de diciembre}

GICOR, Grupo de Investigación en Oncología Radioterápica, Madrid

Miembros AERO implicados: Dr. Manuel de las Heras, presidente de AERO (Hospital Universitario Virgen de la Arrixaca, Murcia), Dra. Ana Mañas, Vicepresidenta de AERO (Hospital Universitario 12 de Octubre, Madrid) y Dr. Jordi Giralt, secretario de la Junta de AERO (Hospital General de la Vall d'Hebrón, Barcelona) 